\title{
Autonomic dysfunction: a novel neurological phenotype associated with Zika virus infection?
}

\author{
Francisco Javier Carod-Artal ${ }^{1,2}$
}

Received: 22 February 2018 / Accepted: 24 February 2018 / Published online: 2 March 2018

c) Springer-Verlag GmbH Germany, part of Springer Nature 2018

Zika virus (ZIKV) is an emergent flavivirus transmitted by the Aedes mosquitoes. Although ZIKV was initially isolated in Uganda in the 1950s, sporadic cases were anecdotally reported in the following decades in a number of African and Asian countries. In the 21st century, there were ZIKV outbreaks in Micronesia (Yap Island, 2007), French Polynesia (2013-2014), and other Pacific islands before the virus eventually arrived in the Americas, with outbreaks in 2015-2017. In February 2016, the World Health Organization declared the ZIKV outbreak a Public Health Emergency of International Concern after a significant increase in microcephaly and Guillain-Barre syndrome (GBS) cases associated with ZIKV infection [1] (Fig. 1).

Around $80 \%$ of ZIKV-infected people do not have symptoms, while symptomatic patients may present with cold-like symptoms, such as fever, itchy rash, non-purulent conjunctivitis and muscle and joint pain. Some patients also report headache and retro-orbital pain, nausea and limb edema. The disease is usually mild and self-limiting, and most subjects become asymptomatic within a couple of weeks. The spectrum of neurological complications associated with ZIKV is increasing, with reported cases of myelitis, encephalitis, acute disseminated encephalomyelitis, encephalomyelitis and peripheral neuritis. A causal link has been established between microcephaly/ZIKV congenital syndrome and ZIKV infection [1]. GBS is the most frequently described neurological complication in adults following ZIKV infection [2]. Recently, case reports of ZIKV-associated sensory polyneuropathy and sensory neuronopathy have been published [3]. Other flaviviruses, such as Dengue, West Nile virus and Japanese encephalitis virus, have well-described

Francisco Javier Carod-Artal

fjcarod-artal@hotmail.com; javier.carodartal@nhs.net

1 Neurology Department, Raigmore Hospital, NHS Highlands, Old Perth Road, IV2 3UJ Inverness, Inverness-shire, UK

2 International Master in Tropical Neurology, International University of Catalonia (UIC), Barcelona, Spain neurotropism, and the tick-borne encephalitis virus appears to have specific tropism for the enteric nervous system $[4,5]$.

In this issue of Clinical Autonomic Research, Rodriguez and colleagues from Colombia reports their study on autonomic symptoms in 35 subjects who had an acute ZIKV infection an average of 63 weeks before the start of the study, and 105 age- and sex-matched controls [6]. The presence of autonomic symptoms was evaluated with the Composite Autonomic Symptom Scale (COMPASS) questionnaire. Subjects with previous history of ZIKV infection had significantly higher COMPASS scores, with a higher burden of symptoms related to orthostatic intolerance, secretomotor function and bladder symptoms.

These are thought-provoking findings. However, this work has two main limitations. First and foremost, the results need to be replicated during the acute and early convalescent stages of ZIKV infection. Moreover, it would be interesting to confirm an association between long-term autonomic dysfunction during the convalescent period and ZIKV infection. The ZIKV viremic period usually lasts around 7 days after the onset of symptoms. However, persistent ZIKV shedding has been detected in some patients in fluids and secretions, notably in urine, semen and vaginal secretions, and the biological and clinical relevance of this fact is unclear [7]. For this reason, ZIKV-infected patients should be evaluated for the presence of autonomic symptoms in the acute setting and at regular follow-up periods to define the natural history and prevalence of ZIKV-related autonomic dysfunction.

The second major limitation is that Rodriguez and colleagues did not perform objective autonomic testing. Because questionnaires of autonomic symptoms may have low specificity to detect true autonomic dysfunction-and may, instead, detect the burden of unspecific symptoms [8]—objective autonomic testing is required to confirm these findings in ZIKV-infected individuals. Autonomic function tests including a tilt-table test, $\mathrm{R}-\mathrm{R}$ interval during deep breathing, Valsalva maneuver, plasma catecholamine levels and sudomotor testing are standard procedures to analyze the 
Fig. 1 World regions with risk of Zika virus infection (January 2018) Adapted from the U.S. Centers for Disease Control and Prevention (https://www.cdc. gov/zika/index.html)

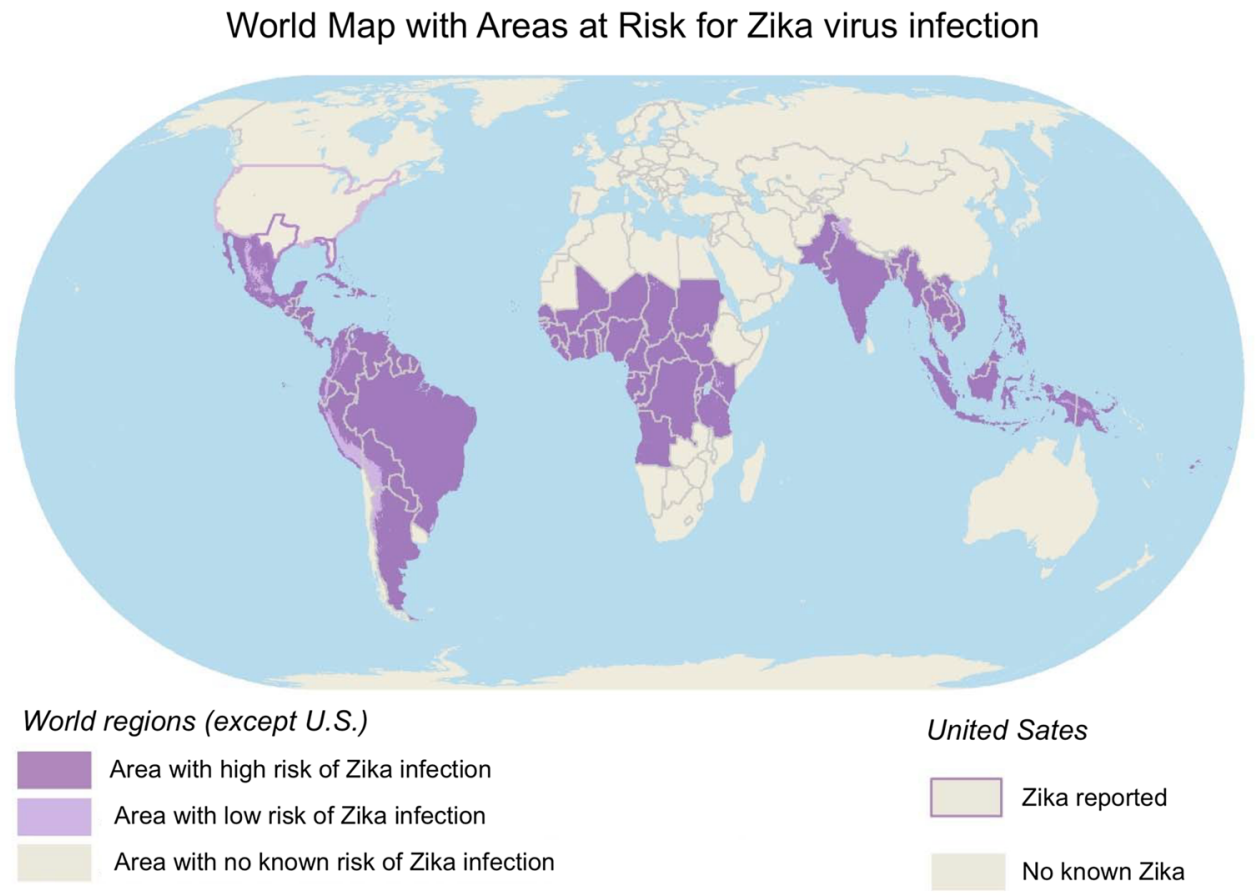

autonomic nervous system. Moreover, patient-centered tools that evaluate symptoms suggesting autonomic dysfunction, like the COMPASS, should be cross-culturally adapted and validated when applied in different cultural and linguistic settings [9]. The validity, reliability, precision of the Spanish version and other important psychometric properties should be evaluated, and mean scores of domains should be compared with those results obtained from other objective measures of autonomic dysfunction (construct convergent validity).

A methodological principle of causality is needed. It cannot be concluded that the observed COMPASS scores obtained almost 1 year after ZIKV infection are necessarily related or caused by the previous ZIKV infection. Other variables (e.g. comorbidities, chronic fatigue and the effect of other unknown or uncontrolled factors) could account for the high COMPASS scores. Nevertheless, it is indeed biologically plausible that ZIKV may induce autonomic dysfunction. Several pathogenic mechanisms have been proposed to explain why a viral infection can cause autonomic dysfunction [10], including invasion of the central nervous system and the direct viral, toxin-mediated or immune-mediated involvement of the peripheral and autonomic nervous system. Also, autonomic dysfunction may happen within the context of a viral myelitis or a post-infectious GBS. Autonomic dysfunction is a wellknown complication in GBS. However, the pathogenesis of a "pure" autonomic dysfunction in patients with ZIKV infection, in the absence of myelitis or GBS, is unknown. The ZIKV-infected patients described by Rodriguez and colleagues apparently did not have any signs or symptoms suggestive of their suffering any other neurological complication associated with ZIKV infection-a fact which creates more questions than answers. So, could ZIKV have a direct neurotropic effect on autonomic neurons? Why may orthostatic tolerance, sudomotor and bladder functions be selectively impaired in ZIKV-infected subjects? As the authors acknowledge, the potential mechanism to explain the association between ZIKV and autonomic symptoms remain unclear.

In murine cultures, ZIKV persistently infected adult sensory neurons of trigeminal and dorsal root ganglia responsible for the innervations of facial mucosa and glands, and the genitourinary tract, respectively [7]. In this in vitro model, autonomic neurons innervating these areas were not susceptible to infection; however, ZIKV infection did induce the destruction of satellite glial cells supporting sensory and autonomic neurons [7]. Sympathetic ganglia neurons can be affected during flavivirus infections such as West Nile virus and could cause autonomic instability. Hypotension and cardiac arrhythmias have been reported in some patients with acute West Nile virus infection [11].

If autonomic nerve damage during the acute ZIKV infection in the absence of other neurological manifestations is confirmed, early diagnosis will become key for the adequate treatment of autonomic dysfunction. Unfortunately, there are still many gaps in our knowledge regarding the full spectrum of neurological manifestations of ZIKV infection, and further research in this area should be included in the agenda of priorities for clinicians. 
Funding None.

\section{Compliance with ethical standards}

Conflict of interest The authors declare no competing financial interest.

\section{References}

1. Pan American Health Organization (PAHO) (2016) Guidelines for surveillance of Zika virus disease and its complications. PAHO, Washington, DC

2. Cao-Lormeau VM, Blake A, Mons S, Lastere S, Roche C, Vanhomwegen J et al (2016) Guillain-Barré syndrome outbreak associated with Zika virus infection in French Polynesia: a case-control study. Lancet 387:1531-1539

3. Martinez ARM, Costa MCM, Novaes MAC, Lima HC, Nucci A, França MC Jr (2017) A novel phenotype of Zika virus-related neurological disease: sensory neuronopathy. Muscle Nerve 57:E100-E101

4. Carod-Artal FJ (2017) The enteric nervous system: another forgotten autonomic target in viral infections? Clin Auton Res $27: 137-138$
5. Versace V, Gutmann B, Sebastianelli L, Martignago S, Saltuari L, Nardone R, Wiedermann CJ (2017) Tick-borne viral encephalomyeloradiculitis complicated by severe autonomic myenteric involvement resulting in irreversible adynamic ileus. Clin Auton Res 27:205-207

6. Rodriguez Y, Rojas M, Ramírez-Santana C, Acosta-Ampudia Y, Monsalve DM, Anaya JM (2018) Autonomic symptoms following Zika virus infection. Clin Auton Res. https://doi.org/10.1007/ s10286-018-0515-1

7. Swartout K, Zlotnick MG, Saver AE, McKenna CM, Bertke AS (2017) Zika Virus persistently and productively infects primary adult sensory neurons in vitro. Pathogens. https://doi.org/10.3390/ pathogens6040049

8. Robinson-Papp J, Sharma SK, George MC, Simpson DM (2017) Assessment of autonomic symptoms in a medically complex, urban patient population. Clin Auton Res 27(1):25-29

9. Carod-Artal FJ, da Silveira Ribeiro L, Kummer W, MartinezMartin P (2009) Psychometric properties of the SCOPA-AUT Brazilian Portuguese Version. Mov Disord 25:205-212

10. Carod Artal FJ (2018) Infectious diseases causing autonomic dysfunction. Clin Auton Res 28:67-81

11. Bode AV, Sejvar JJ, Pape WJ, Campbell GL, Marfin AA (2006) West Nile virus disease: a descriptive study of 228 patients hospitalized in a 4-county region of Colorado in 2003. Clin Infect Dis 42:1234-1240 